\title{
Are island and mainland biotas different? Richness and level of generalism in parasitoids of a microlepidopteran in Macaronesia
}

\author{
Ana M. C. Santos, Colin Fontaine, Donald L. J. Quicke, Paulo A. V. Borges and Joaquín Hortal \\ A. M. C. Santos(ana.margarida.c.santos@googlemail.com) and D. L. J. Quicke, Dept of Biology, Imperial College London, Silwood Park \\ Campus, Ascot, Berkshire, SL5 7PY, UK. DLJQ also at: Dept of Entomology, Natural History Museum, Cromwell Road, London, SW7 5BD, \\ UK. - P. A. V. Borges and AMCS, Dep. de Ciências Agrárias - CITA-A (Azorean Biodiversity Group), Univ. dos Açores, Rua Capitão Joäo de \\ Ávila, PT-9700-042 Angra do Heroísmo, Portugal. - C. Fontaine, UMR 7204, Conservation des Espèces, Restauration et Suivi Populations, \\ MNHN, UPMC, CNRS, FR-75005 Paris, France. - J. Hortal and CF, Centre for Population Biology, Imperial College London, Silwood Park \\ Campus, Ascot, Berkshire, SL5 7PY, UK. JH also at: Dept. Biodiversidad y Biología Evolutiva, Museo Nacional de Ciencias Naturales (CSIC), \\ C/José Gutiérrez Abascal 2, ES-28006 Madrid, Spain.
}

\begin{abstract}
Island communities are exposed to several evolutionary and ecological processes that lead to changes in their diversity and structure compared to mainland biotas. These phenomena have been observed for various taxa but not for parasitoids, a key group in terms of community diversity and functioning. Here we use the parasitoid communities associated with the moth Acroclita subsequana (Lepidoptera: Tortricidae) in the Macaronesian region, to test whether species richness differs between islands and mainland, and whether island parasitoid faunas are biased towards generalist species. Host larvae were collected on several islands and adjacent mainland, carefully searched for ectoparasitoid larvae and dissected to recover any endoparasitoids. Parasitoids were classified as idiobionts, which usually have a wide host range (i.e. generalists), or koinobionts that are considered specialists. Mainland species richness was lower than expected by chance, with most of the species being koinobionts. On the other hand, island communities showed a greater proportion of idiobiont species. Overall parasitism rates were similar between islands and mainland, but islands had higher rates of parasitism by idiobionts than expected by chance, and mainland areas showed the highest koinobiont parasitism rates. These results suggest that island parasitoid communities are dominated by generalists, in comparison to mainland communities. Several hypotheses may explain this pattern: (1) generalist parasitoids might have better dispersal abilities; (2) they may be less constrained by 'sequential dependencies'; and (3) island parasitoids probably have fewer competitors and/or predators, thus favouring the establishment of generalists. New studies including multiple hosts, other habitats, and/or more islands are necessary to identify which of these processes shape island parasitoid communities.
\end{abstract}

Island faunas tend to be species-poor and disharmonic in relation to the mainland (i.e. continental areas), and this is especially the case for oceanic islands (Williamson 1981, Gillespie and Roderick 2002, Whittaker and FernándezPalacios 2007). Typically, islands have fewer species than an area of similar size on the mainland, which often results in some functional groups (i.e. trophic or ecological guilds) being missing or underrepresented in their communities (Whittaker and Fernández-Palacios 2007). In addition to these differences in the species pool, the feeding interactions among species often vary as well. Following a founding event, several evolutionary and ecological processes take place (Losos and Ricklefs 2009), including ecological release, density compensation, niche expansion and niche shifts (Gillespie and Roderick 2002, Parent and Crespi 2009). These processes are promoted by factors such as the existence of empty or invasible niche space, low interspecific competition and lack of entire groups of predators, parasitoids or pathogens, which usually contribute to increase the number of species using a broader range of resources (Whittaker and Fernández-Palacios 2007). In other words, oceanic islands tend to host more generalist species than their source mainlands, both because generalists may have an a priori advantage during the colonization process (Piechnik et al. 2008), and because some species are able to increase their niche width after reaching a new territory (Schlotfeldt and Kleindorfer 2006). This tendency for island populations to have wide ecological niches seems to be a general pattern and has been observed at least in birds (Diamond 1970, Olesen and Valido 2004, Schlotfeldt and Kleindorfer 2006), bryophytes (Gabriel and Bates 2005), lizards (Scott et al. 2003, Olesen and Valido 2003, 2004) and several insect groups (Olesen et al. 2002, Ribeiro et al. 2005).

Parasitoids are insects whose larvae develop by feeding on (ectoparasitoids) or within (endoparasitoids) an arthropod host, eventually killing it (Eggleton and Belshaw 1992, Godfray 1994, Quicke 1997). Although they are best known from the parasitic Hymenoptera, which account for 
approximately three-quarters of the total number of known parasitoid species, other orders such as Diptera, Coleoptera, Lepidoptera and Neuroptera also include parasitoid species (Eggleton and Belshaw 1992, Godfray 1994). Parasitoids can be divided into two groups, depending on their life history strategies: koinobionts, which allow the host to continue its development after oviposition, and idiobionts, which do not (Askew and Shaw 1986). Many life history traits, including host ranges and mode of parasitism, appear to be correlated with this dichotomy (Sheehan and Hawkins 1991, Hawkins 1994, Quicke 1997, Mayhew and Blackburn 1999).

The host range of a particular parasitoid species is the group of potential hosts that it can usually attack successfully, after exhibiting a pattern of searching behaviour that allows it to find them regularly (Shaw 1994). Koinobionts usually have a narrower host range than idiobionts (Askew and Shaw 1986, Sheehan and Hawkins 1991, Hawkins 1994, Althoff 2003, but see Mills 1992) because they have a more prolonged interaction with their hosts' immune system; therefore, the adaptations needed to overcome this problem are believed to restrict the number of hosts that koinobionts can attack successfully. On the other hand, when attacking larval hosts, idiobionts paralyse their hosts on the moment of oviposition, and their interaction with the host immune system is minimal, allowing them to be physiologically able to develop on a wider range of hosts (Askew and Shaw 1986, Hawkins 1994). Consequently, idiobionts are expected to be able to shift on to novel hosts more readily than koinobionts can (Cornell and Hawkins 1993, Shaw 1994). In the absence of detailed rearing records, the koinobiont/idiobiont dichotomy represents a practical criterion for distinguishing between parasitoids that tend to be specialists (koinobionts) and parasitoids that are potentially more generalists (idiobionts) in terms of the host range attacked (Hawkins et al. 1990).

Realized host range may change over both evolutionary and biological time. Parasitoids can exhibit plasticity in the range of hosts they attack, and thus are able to respond to inconstant and uneven environments (Cornell and Hawkins 1993, Godfray et al. 1995, Hawkins and Marino 1997). Since hosts on islands may be unusual or novel compared to those on the mainland, parasitoids arriving on islands may be forced to attempt to utilize less preferred or novel hosts. Therefore island faunas would be expected to be biased towards generalist species (i.e. idiobionts), at least in the initial stages of their colonization. Although there are some studies on the dispersal, colonization, and establishment of parasitoids in new areas, most focus on the landscape level (Kruess and Tscharntke 2000, van Nouhuys and Hanski 2002, Elzinga et al. 2007). Few studies analyse parasitoids' host ranges (and host shifts) on a wider geographic scale, and those that do usually rely on literature records (Cornell and Hawkins 1993, Hawkins 1994, Hawkins and Marino 1997, Santos et al. (in press), but see Stone et al. 1995). The diversity patterns of parasitoids remain particularly poorly known for oceanic islands, for which the only published works are mostly limited to checklists (Belokobylskij and Maetô 2008, Bennet 2008), and are usually biased towards introduced species and agricultural habitats (Funasaki et al. 1988, Peck et al. 1998, Santos et al. 2005, but see Maetô and Thornton 1993, Schoener et al. 1995, Hodkinson et al. 2004).
Here we study how the diversity and attack strategy of parasitoid communities associated with the moth Acroclita subsequana (Lepidoptera: Tortricidae) feeding on spurges (Euphorbia spp., Euphorbiaceae) vary between the islands and adjacent mainland of the Macaronesian region. This study system provides an opportunity to investigate how parasitoid communities change geographically, because it consists of populations of a single host scattered throughout a region for which biogeographical patterns are well known (Triantis et al. 2010). We specifically test whether for a given host: (1) parasitoid species richness differs between island and mainland territories; (2) the island parasitoid communities are biased towards generalist species (i.e. islands have a higher proportion of idiobiont species); and (3) whether these changes in composition and diversity of the parasitoid communities translate into variations in parasitism rates.

\section{Material and methods}

\section{Study area}

This study was conducted in four islands and three areas of the adjacent mainland from the Macaronesian region (northeastern Atlantic). Larvae of the moth Acroclita subsequana (Lepidoptera: Tortricidae) found feeding on spurges (Euphorbia spp.) were collected from 55 study sites located in the islands of Madeira, La Gomera, La Palma and Tenerife, as well as in Morocco (western and northern regions) and mainland Portugal (Fig. 1, Table 1).

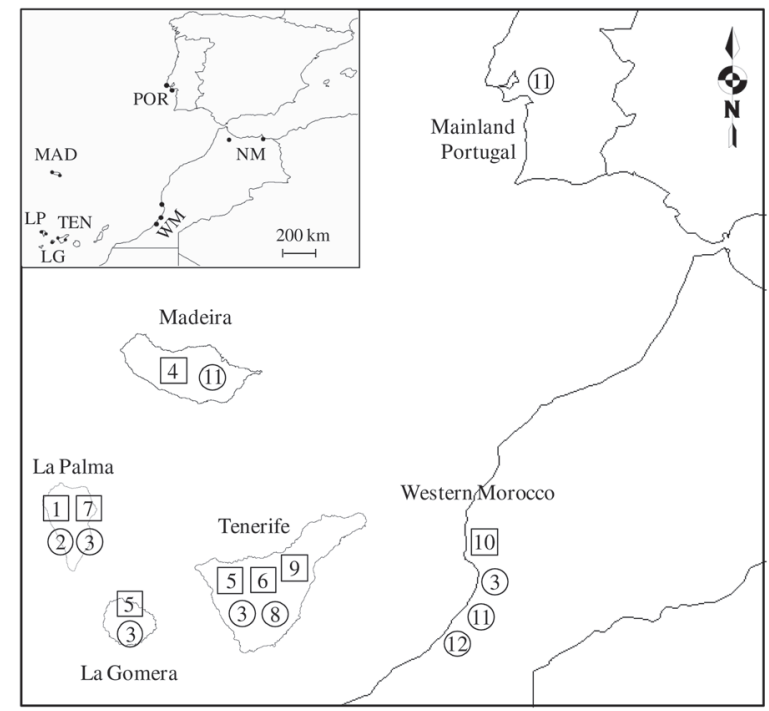

Figure 1. Geographical distribution of the parasitoid species found in the studied islands and mainland areas of Macaronesia. Squares represent idiobionts and circles are koinobionts. Numbers correspond to the code given to each parasitoid MOTU (see Santos et al. 2011 for more information). The scale of the islands and the distance between them and the mainland are modified for the ease of visualization. The map in the inset is correctly scaled, and shows the actual position of each island and the location of the different sampling areas (represented by the black dots). MAD - Madeira Island; LG - La Gomera; LP - La Palma; TEN - Tenerife; WM - western Morocco; NM - northern Morocco; POR - mainland Portugal. 
Table 1. Abundance and species richness of hosts and parasitoids collected on each study area.

\begin{tabular}{|c|c|c|c|c|c|c|c|}
\hline Area & No. of sites & Host identifier & No. of hosts & Idiobiont species & Koinobiont species & No. of idiobionts & No. of koinobionts \\
\hline \multicolumn{8}{|l|}{ Islands } \\
\hline Madeira & 5 & hMOTU13 & 57 & 1 & 1 & 6 & 2 \\
\hline La Gomera & 8 & hMOTU14 & 180 & 1 & 1 & 6 & 6 \\
\hline La Palma & 7 & hMOTU15 & 187 & 2 & 2 & 6 & 5 \\
\hline Tenerife & 9 & hMOTU16 & 240 & 3 & 2 & 10 & 3 \\
\hline \multicolumn{8}{|l|}{ Mainland } \\
\hline W Morocco & 16 & hMOTU17 & 472 & 1 & 3 & 6 & 20 \\
\hline N Morocco & 4 & hMOTU18 & 92 & 0 & 0 & 0 & 0 \\
\hline Portugal & 6 & hMOTU18 & 187 & 0 & 1 & 0 & 9 \\
\hline
\end{tabular}

Number of sites indicates the number of sites sampled per study area; Host identifier is the code given to each host molecular operational taxonomic unit (MOTU) collected from the study areas (see more details in Santos et al. 2011). No. of hosts is the total number of Acroclita subsequana larvae collected. Idiobiont species and koinobiont species correspond to the number of idio- and koinobiont MOTUs, respectively, and no. of idiobionts and no. of koinobionts are the number of host larvae that were attacked by idio- or koinobionts, respectively.

\section{Sampling and species identification}

A detailed description of the sampling protocol and identification methods is presented in Santos et al. (2011). Briefly, tortricid larvae protected by flowers, fruits, leaves and stems that they roll or tie together, or partly concealed within the stems or fruit's capsule, were collected by hand during about one hour per sampling site ( 15 minutes on sites with relatively few Euphorbia sp. individuals; no. of host larvae collected in Table 1). All specimens were then preserved in ethanol and stored below $5^{\circ} \mathrm{C}$ until they were dissected in order to find ectoparasitoid and/or endoparasitoid larvae. To avoid spurious effects related with host larvae age, specimens with a body size length lower than approx. $1 \mathrm{~cm}$ were not considered. Both hosts and parasitoids were sequenced for a ca 650 base-pair $5^{\prime}$ fragment of the mitochondrial cytochrome oxidase I gene (COI), and assigned to molecular operational taxonomic units (MOTUs) based on pairwise genetic distances and treebased and similarity-based methods. Parasitoids were grouped into 12 MOTUs belonging to the Hymenoptera (Bethylidae, Braconidae, Ichneumonidae and Chalcidoidea) and Diptera (Cecidomyiidae and Tachinidae) (see Table 2 in Santos et al. 2011). These MOTUs were assumed to correspond to different parasitoid species, that were easily classified as idio- or koinobionts according to the known biology of the families and subfamilies they belong to (Santos et al. in press). Specimens that were not sequenced or were only identified to superfamily level (e.g. Chalcidoidea), were classified according to the attack strategy observed during the dissection process: ectoparasitoids were classified as idiobionts and endoparasitoids were assumed to be koinobionts (following Hawkins 1994 and Mayhew and Blackburn 1999). Hosts comprised six MOTUs, each one found in one of the sampled areas, except for northern Morocco and mainland Portugal that share the same host MOTU (Table 1). Although these host MOTUs can potentially correspond to different cryptic species within the currently valid species $A$. subsequana (Santos et al. 2011), for the purpose of this study they can be considered the same (or very similar) type of resource.

\section{Statistical analyses}

When the same sampling effort with standardized techniques is carried out in different areas and/or community types, sampling success may not always be the same and may lead to important biases in the total species richness inventoried at each site. Therefore, in such situations it may be necessary to use extrapolation techniques to 'standardize' richness data (Hortal et al. 2006). Here, parasitoid species richness was estimated using non-parametric estimators. Given that different extrapolation methods provide different richness estimates we adopted the more conservative strategy of using several of them, namely: Incidence-based coverage estimator (ICE), Chao's incidence-based estimator (Chao2), 1st order Jackknife (Jack1), 2nd order Jackknife (Jack2) and Michaelis-Menten (MM) (see Colwell and Coddington 1994, Gotelli and Colwell 2001, Hortal et al. 2006 for more details on these estimators and their performance). All these calculations were done in EstimateS 8.2.0 software (Colwell 2009), randomising the order of the samples 1000 times.

Table 2. Comparison between observed and randomised parasitoid species richness and parasitism rate of each study area.

\begin{tabular}{lcccccc}
\hline & Madeira & La Gomera & La Palma & Tenerife & Western Morocco & Mainland Portugal \\
\hline Total parasitoid species richness & + & -+ & - & - & $-^{*}$ & $-^{* *}$ \\
Idiobiont species richness & + & - & - & - & $-^{* *}$ & $--^{*}$ \\
Koinobiont species richness & + & - & + & + & + & - \\
Total parasitism rate & $++^{* *}$ & + & + & - & + & - \\
Idiobiont parasitism rate & $+* *$ & + & + & $+^{+}$ & - & + \\
Koinobiont parasitism rate & + & + & - & $-^{+}$ & + & +
\end{tabular}

Cases where observed results are lower than the median of the null expectation are represented by (-). Cases where observed results are higher than the median of the null expectation are represented by $(+)$. Cases outside the $95 \% \mathrm{Cl}$ (i.e. significantly different from the overall pool) are represented by ${ }^{*} p<0.05 ;{ }^{* *} p<0.01$, while those that are marginally significant $(p<0.1)$ are represented by ${ }^{\dagger}$. Randomization results are presented in Supplementary material Fig. S1-S6. 
We used a bootstrap procedure to evaluate whether observed species richness and parasitism rates (i.e. number of parasitoid individuals per number of hosts collected) differed from what could be expected by chance. For a given locality where $\mathrm{N}$ caterpillars were collected, the expected null distribution was created by resampling the total dataset with replacement to create 5000 samples of the same size. Parasitoid richness and parasitism rates of both idio- and koinobionts were calculated for each of these samples to obtain the distribution of random expectations. Observed values were then compared to these distributions, and were considered significantly different from the null expectation when falling outside the $95 \%$ confidence interval. We also report the marginally significant results $(p<0.1)$ because of the low power of our randomization analyses for detecting differences in community structure (based on just 79 parasitized caterpillars out of 1415 randomized ones). These analyses were performed using a script written in $\mathrm{R}$ (R Development Core Team 2009).

\section{Results}

A total of 1415 Acrolita subsequana larvae were collected and dissected (Table 1). Seventy-nine larvae were attacked by one (or more) parasitoids. Parasitoids attacking the same host larvae always belonged to the same species, so we treated them as a single parasitoid attack. Sixty-five out of these 79 parasitoid larvae $(82.3 \%)$ were successfully sequenced and assigned to 12 MOTUs (Table 2 in Santos et al. 2011). In total, 34 parasitoid larvae were classified as idiobionts and 45 were considered to be koinobionts (Table 1).

Mainland species richness was significantly lower than expected by chance (Table 2, Supplementary material Fig. S1). Islands as a whole had more than twice as many species than observed in all mainland areas. When the study sites were compared, Tenerife was the richest, followed by La Palma and western Morocco. Mainland Portugal had the lowest richness and northern Morocco was the only region where no parasitoids were found (Fig. 1, Table 1). Due to this, the latest area will not be mentioned any further in this work. Estimated species richness values differed from the observed ones (Table 3), with the Chao 2 estimates being the most similar to the observed data, while Jack2 usually showed higher values. Tenerife was estimated to be the study area with the most parasitoid species, while mainland Portugal was the one with the least (Fig. 2, Table 3).

A greater proportion of idiobiont species was found in Tenerife, while western Morocco had the highest number of koinobiont species (Fig. 1); no idiobionts were found in mainland Portugal. Two koinobiont species were found on both island and mainland areas, while there were no shared idiobionts between these two types of study areas. Although more idiobiont species were detected on islands (only one species was found on the mainland) (Fig. 1), there was no significant difference between the number of idio- and koinobiont species found on island and mainland study areas $\left(\chi^{2}=1.4\right.$; 1 DF; $\mathrm{p}=0.237$ ). Nevertheless, while observed idiobiont richness from western Morocco and mainland Portugal was significantly lower than the correspondent null expectation of bootstrapped values (Table 2, Supplementary material Fig. S2), there were no significant differences between koinobiont richness and the corresponding null expectation (Table 2, Supplementary material Fig. S3).

The overall parasitism rate across study areas was 5.6\%. Although the proportion of host larvae attacked was slightly higher on the islands than on mainland, this difference was not significant $\left(\chi^{2}=2.584 ; 1 \mathrm{DF} ; \mathrm{p}=0.108\right)$. The highest parasitism rate $(14.0 \%)$ was found on Madeira, this value being significantly higher than the null expectation (Table 2, Supplementary material Fig. S4). On the contrary, mainland Portugal showed the lowest parasitism rate $(4.8 \%)$.

The ratio of number of larvae parasitized by idiobionts to those parasitized by koinobionts differed significantly between islands and mainland (Table 4). The highest parasitism rate by idiobionts was found in Madeira (10.5\%), and the lowest (apart from mainland Portugal) was registered in western Morocco (1.3\%; Table 1). Conversely, the attack rate by koinobionts was highest in mainland Portugal (4.8\%) and lowest in Tenerife (1.3\%). Observed idiobiont parasitism rates from Madeira and Tenerife were either significantly or marginally significantly higher than the correspondent null expectation, while in mainland Portugal observed idiobiont parasitism rate was significantly lower

Table 3. Observed (Sobs) and estimated parasitoid species richness in each study area.

\begin{tabular}{|c|c|c|c|c|c|c|c|c|}
\hline & Uniques & Duplicates & Sobs & ICE & Chao2 $\pm \mathrm{SD}$ & Jack1 $\pm \mathrm{SD}$ & Jack2 \pm SD & MM \\
\hline Madeira & 1 & 0 & 2 & 3.11 & $2 \pm 0.35$ & $2.98 \pm 0.98$ & $3.95 \pm 0$ & 2.57 \\
\hline La Gomera & 1 & 0 & 2 & 3.11 & $2 \pm 0.35$ & $2.99 \pm 0.99$ & $3.98 \pm 0$ & 3.96 \\
\hline La Palma & 1 & 2 & 4 & 4.50 & $4 \pm 0.17$ & $4.99 \pm 0.99$ & $4.02 \pm 0$ & 7.43 \\
\hline Tenerife & 2 & 1 & 5 & 6.65 & $5.5 \pm 1.29$ & $6.99 \pm 1.41$ & $7.99 \pm 0$ & 8.51 \\
\hline W Morocco & 1 & 0 & 4 & 4.41 & $4 \pm 0.43$ & $5 \pm 1$ & 5.99 & 4.69 \\
\hline M Portugal & 0 & 0 & 1 & 1.00 & $1 \pm 0.01$ & 1 & $1 \pm 0$ & 1.17 \\
\hline All islands & 3 & 2 & 10 & 11.70 & $11 \pm 1.82$ & $13 \pm 1.73$ & $14 \pm 0$ & 14.44 \\
\hline All mainland & 1 & 0 & 4 & 4.59 & $4 \pm 0.43$ & $5 \pm 1$ & 6 & 4.39 \\
\hline All sites & 3 & 2 & 12 & 13.65 & $13 \pm 1.82$ & $15 \pm 1.73$ & $16 \pm 0$ & 14.45 \\
\hline
\end{tabular}

Uniques and duplicates are the number of parasitoid species that occur in only one or two samples (i.e. host larvae), respectively. ICE, Chao2, Jack1, Jack2 and $\mathrm{MM}$ correspond to the different species richness estimators used (incidence-based coverage estimator, Chao's incidence-based estimator, Jackknife 1, Jackknife 2 and Michaelis-Menten, respectively; see Colwell 2009 and Material and methods for further information). When relevant, the standard deviations of the estimations (SD) are shown next to the estimated values. M Portugal corresponds to mainland Portugal. All islands corresponds to all islands as a whole; All mainland to all mainland areas; and All sites corresponds to all study areas. Northern Morocco not shown due to the lack of recorded parasitoids. 


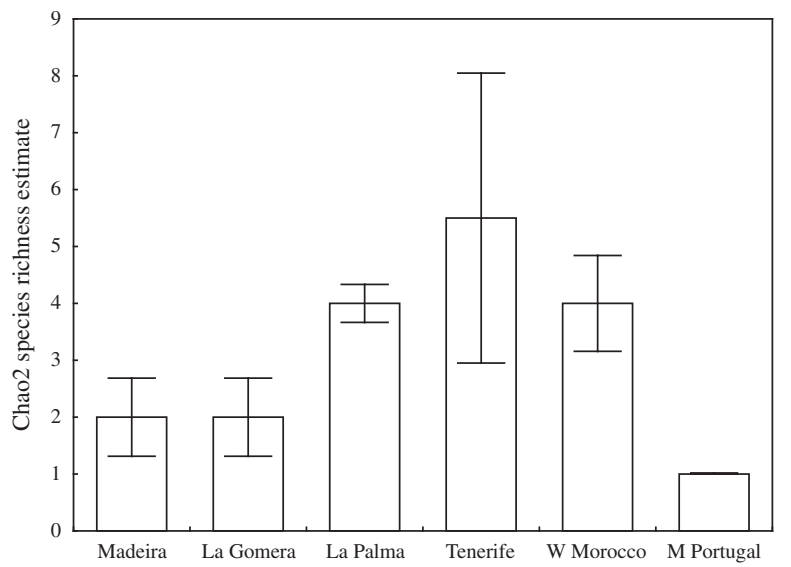

Figure 2. Parasitoid species richness in each study area, as estimated by Chao2 (see also Table 3). Bars correspond to the 95\% CI. M Portugal corresponds to mainland Portugal.

than the null expectation (Table 2, Supplementary material Fig. S5). Finally, Tenerife, and to a lesser extent La Palma, were the only islands where observed koinobiont parasitism rates departed from the null expectation, although such deviation was only marginally significantly lower in the case of Tenerife (Table 2, Supplementary material Fig. S6).

\section{Discussion}

Islands commonly have fewer species than apparently comparable mainland areas (MacArthur et al. 1972, Rosenzweig 1995, Gillespie and Roderick 2002, Whittaker and FernándezPalacios 2007). Apart from isolation (MacArthur and Wilson 1967), another reason for this pattern might be that the ability of many species to colonize and survive on islands is constrained by the lack of suitable host/food resources (Holt et al. 1999). Contrary to this expectation, the island and mainland areas studied showed comparable richness values; La Palma had a similar number of parasitoid species to western Morocco, while Tenerife was even richer.

We also found that the strength of the host-parasitoid interaction, measured as parasitism rate, was slightly, though not significantly, higher on islands. The highest parasitism rate was registered for Madeira Island, which was also one of the studied areas with the lowest species richness. Rodriguez and Hawkins (2000) and Connahs et al. (2009) similarly found higher rates of parasitism associated with lower parasitoid diversity for Great Britain and Costa Rica, Ecuador and Panama, respectively (but see Tylianakis et al. 2006).
Although the overall parasitism rates were similar on islands and mainland, their community structure differed. Our results indicate that island parasitoid communities may be biased in favour of idiobiont species, which are considered to be generalists, whereas mainland communities seem to be dominated by koinobionts, which are thought to be more specialised. The lack of species specialised in attacking A. subsequana on the islands could be compensated for by the higher number of presumed generalists that are potentially able to feed on a higher number of hosts. This suggests that if our survey encompassed a larger number of host species, the total parasitoid species richness would have been smaller on islands, since numerous host species would be parasitized by a reduced group of generalist parasitoid species.

Although the trend for island populations to be more generalist (in terms of either habitat or feeding niche) then their mainland counterparts has already been suggested for many taxa (Diamond 1970, Olesen and Valido 2003, 2004, Schlotfeldt and Kleindorfer 2006, Whittaker and Fernández-Palacios 2007), as far as we know this is the first time that this pattern has been observed for parasitoids. Several hypotheses may be formulated to explain the tendency for parasitoid faunas to be composed by more generalist species on islands. First, generalists may simply be better dispersers than specialists. However, although it is known that parasitoids can disperse on the scale of kilometres (Antolin and Strong 1987, Godfray 1994, Jones et al. 1996), and that dispersal ability varies between species (van Nouhuys and Hanski 2002, Elzinga et al. 2007), very little is known about the eventual existence of a relationship between dispersal ability and the potential host range of the parasitoids. A second alternative hypothesis would be that generalist parasitoids colonize islands before specialists because they are less constrained by 'sequential dependencies' than specialists (Holt et al. 1999), being more likely to be able to consume any early-arriving hosts (Piechnik et al. 2008). However, the level of generalism on island and mainland parasitoid communities has never been compared before (but see Santos et al. in press); the few available evidences are contradictory, indicating either that island parasitoid faunas are biased towards koinobionts (Maetô and Thornton 1993), that no ecological or biological factors correlate with the probability of colonizing new hosts (Godfray et al. 1995, Hawkins and Marino 1997), or that generalists can more readily include new hosts on their host range (Cornell and Hawkins 1993). Here it is important to take into account that these studies were carried out in very recent communities that are probably not in equilibrium. Given that the time required for the evolution

Table 4. Pairwise comparisons of the number of host larvae parasitized by idio- and koinobionts between study areas. Values correspond to the results of $\chi^{2}$ analyses $\left(\mathrm{H}_{0}=\right.$ no differences in the proportions of larvae parasitized on each study area).

\begin{tabular}{|c|c|c|c|c|c|}
\hline & Madeira & La Gomera & La Palma & Tenerife & W Morocco \\
\hline La Gomera & 1.25 & & & & \\
\hline La Palma & 0.83 & 0.05 & & & \\
\hline Tenerife & 0.01 & 1.96 & 1.34 & & \\
\hline W Morocco & $7.22^{* *}$ & $2.75^{\mathrm{a}}$ & $3.49^{b}$ & $10.39^{* *}$ & \\
\hline Mainland Portugal & $10.43^{* *}$ & $6.30^{*}$ & $7.01^{* *}$ & $12.69^{* * *}$ & 2.51 \\
\hline
\end{tabular}

${ }^{*} p<0.05 ;{ }^{* *} p<0.01 ;{ }^{* * *} p<0.001 ;{ }^{a} p=0.097 ;{ }^{b} p=0.062$. 
and full acquisition of parasitoids by some hosts may fall between 100 and 10000 years (Cornell and Hawkins 1993), it is difficult to extend these interpretations to our study system. Finally, a third hypothesis for explaining the apparent preponderance of generalist parasitoids on the Macaronesian islands would be ecological release, a phenomenon that is typical in many island populations (Diamond 1970, Olesen et al. 2002, Scott et al. 2003, Whittaker and Fernández-Palacios 2007). When a species colonizes an island it often encounters a new environment in which competitors and predators are absent, being therefore able to exploit a wider niche space, which in turn leads to niche expansion and/or niche shifts (Cox and Ricklefs 1977).

Unfortunately, our data do not allow a formal test of any of these hypotheses. First, we focused on the parasitoid communities of a single host species, and therefore we have no information on the complete host range of each parasitoid species. Second, we studied only larval parasitoids, which might be different from parasitoids attacking hosts on their egg or pupal state. Third, given that the host studied lives in a semiconcealed environment (rolled in leaves, buds or flowers) it is difficult to generalize to other types of hosts, such as exposed free-living caterpillars or concealed larvae (e.g. wood borers). As the parasitoids attacking these two types of larvae tend to either be koinobionts or idiobionts, respectively (Askew and Shaw 1986, Hawkins 1994, Quicke 1997), the assembly of parasitoid communities is probably different from that of semiconcealed hosts. Finally, although the slopes of the species accumulation curves indicate that the sampling effort was appropriate to provide a reliable inventory of the diversity of each study area (Table 3 in Santos et al. 2011), the total number of parasitoid individuals collected on each area is still small (here, note that increasing such numbers would involve a costly large-scale research project).

In spite of these drawbacks, we showed that for a particular host species, island parasitoid communities are at least as species rich as those found on the mainland, but that the species composition changed from communities dominated by specialists in the mainland to communities dominated by generalists on islands. These patterns are probably the outcome of several interacting processes, some of which we discussed above. Further work is yet necessary to unveil the causes of the higher numbers of idiobionts associated with A. subsequana on the Macaronesian islands in relation to the mainland. Expanding this type of approach to multiple hosts, or other habitats and islands, will provide further insights into the ecology of parasitoid communities, and ultimately to the understanding of the processes shaping species interactions in a biogeographical context.

Acknowledgements - We want to thank Ángel Fernández, Cristina Fuentes, Félix Medina, Cristina Padrón, Ângel Palomares, Pedro Romero and Marina Sequeira for helping us obtain the fieldwork permits, and Franquinho Aguiar, Isabel Amorim, Mário Boieiro, Raimundo Cabrera, Abdelhaq Hanafi, Alberto Jiménez-Valverde, Jorge Lobo, António Machado, Glória Ortega, Fernando Pereira and Carla Rego for providing support during field work. Also, we acknowledge Rebecca Fuller, Rebecca Jeffrey and Adila Shaukat for their support in the laboratory, and Guillaume Besnard and Sofia Gabriel for their help with molecular data analyses. Marcos Báez and Ole Karsholt provided specimens of $A$. subsequana, Joaquín
Baixeras identified some of our tortricid specimens and provided adult specimens of $A$. subsequana, and Winnie Halwachs, Daniel Janzen and Alex Smith provided some parasitoid sequences. AMCS was supported by a Portuguese FCT grant (SFRH/ BD/21496/2005) co-funded by the European Social Fund POPHQREN program, and CF and JH by the UK Natural Environment Research Council.

\section{References}

Althoff, D. M. 2003. Does parasitoid attack strategy influence host specificity? A test with New World braconids. - Ecol. Entomol. 28: 500-502.

Antolin, M. F. and Strong, D. R. 1987. Long-distance dispersal by a parasitoid (Anagrus delicatus, Mymaridae) and its host. Oecologia 73: 288-292.

Askew, R. R. and Shaw, M. R. 1986. Parasitoid communities: their size, structure and development. - In: Waage, J. and Greathead, D. (eds), Insect parasitoids. Academic Press, pp. 225264.

Belokobylskij, S. A. and Maetô, K. 2008. Doryctinae (Hymenoptera: Braconidae) of Ogasawara islands (Japan). - Ann. Zool. 58: $125-166$.

Bennett, D. J. 2008. The Ophioninae wasps of Hawaii (Hymenoptera: Ichneumonidae). - J. Hymenopt. Res. 17: 1-43.

Colwell, R. K. 2009. EstimateS, statistical estimation of species richness and shared species from samples. - User's guide and computer program available at $<$ http://viceroy.eeb.uconn. edu/estimates $>$, Univ. of Connecticut, USA.

Colwell, R. K. and Coddington, J. A. 1994. Estimating terrestrial biodiversity through extrapolation. - Phil. Trans. R. Soc. B 345: 101-118.

Connahs, H. et al. 2009. Geographic variation in host-specificity and parasitoid pressure of an herbivore (Geometridae) associated with the tropical genus Piper (Piperaceae). - J. Insect Sci. 9: 28.

Cornell, H. V. and Hawkins, B. A. 1993. Accumulation of native parasitoid species on introduced herbivores: a comparison of hosts as natives and hosts as invaders. - Am. Nat. 141: 847-865.

Cox, G. W. and Ricklefs, R. E. 1977. Species diversity and ecological release in Caribbean land bird faunas. - Oikos 28: 113-122.

Diamond, J. M. 1970. Ecological consequences of island colonization by southwest Pacific birds, I. Types of niche shifts. - Proc. Natl Acad. Sci. 67: 529-536.

Eggleton, P. and Belshaw, R. 1992. Insect parasitoids: an evolutionary overview. - Phil. Trans. R. Soc. B 337: 1-20.

Elzinga, J. A. et al. 2007. Distribution and colonisation ability of three parasitoids and their herbivorous host in a fragmented landscape. - Basic Appl. Ecol. 8: 75-88.

Funasaki, G. Y. et al. 1988. A review of biological control introductions in Hawaii: 1890 to 1985. - Proc. Hawaii. Entomol. Soc. 28: 105-160.

Gabriel, R. and Bates, J. W. 2005. Bryophyte community composition and habitat specificity in the natural forests of Terceira, Azores. - Plant Ecol. 177: 125-144.

Gillespie, R. G. and Roderick, G. K. 2002. Arthropods on islands: colonization, speciation, and conservation. - Annu. Rev. Entomol. 47: 595-632.

Godfray, H. C. J. 1994. Parasitoids: behavioral and evolutionary ecology. - Princeton Univ. Press.

Godfray, H. C. J. et al. 1995. The recruitment of parasitoid species to two invading herbivores. - J. Anim. Ecol. 64: 393-402.

Gotelli, N. J. and Colwell, R. K. 2001. Quantifying biodiversity: procedures and pitfalls in the measurement and comparison of species richness. - Ecol. Lett. 4: 379-391. 
Hawkins, B. A. 1994. Pattern and process in host-parasitoid interactions. - Cambridge Univ. Press.

Hawkins, B. A. and Marino, P. C. 1997. The colonization of native phytophagous insects in North America by exotic parasitoids. - Oecologia 112: 556-571.

Hawkins, B. A. et al. 1990. Influences of host feeding-niche and foodplant type on generalist and specialist parasitoids. - Ecol. Entomol. 15: 275-280.

Hodkinson, I. D. et al. 2004. Invertebrate community assembly along proglacial chronosequences in the high Arctic. - J. Anim. Ecol. 73: 556-568.

Holt, R. D. et al. 1999. Trophic rank and the species-area relationship. - Ecology 80: 1495-1504.

Hortal, J. et al. 2006. Evaluating the performance of species richness estimators: sensitivity to sample grain size. - J. Anim. Ecol. 75: 274-287.

Jones, T. H. et al. 1996. Relative movement patterns of a tephritid fly and its parasitoid wasps - Oecologia 106: 317-324.

Kruess, A. and Tscharntke, T. 2000. Species richness and parasitism in a fragmented landscape: experiments and field studies with insects on Vicia sepium. - Oecologia 122: 129-137.

Losos, J. B. and Ricklefs, R. E. 2009. Adaptation and diversification on islands. - Nature 457: 830-836.

MacArthur, R. H. and Wilson, E. O. 1967. The theory of island biogeography. - Princeton Univ. Press.

MacArthur, R. H. et al. 1972. Density compensation in island faunas. - Ecology 53: 330-342.

Maetô, K. and Thornton, I. W. B. 1993. A preliminary appraisal of the braconid (Hymenoptera) fauna of the Krakatau Islands, Indonesia, in 1984-1986, with comments on the colonizing abilities of parasitoid modes. - Jap. J. Entomol. 61: 787-801.

Mayhew, P. J. and Blackburn, T. M. 1999. Does development mode organize life-history traits in the parasitoid Hymenoptera? - J. Anim. Ecol. 68: 906-916.

Mills, N. J. 1992. Parasitoid guilds, life-styles, and host ranges in the parasitoid complexes of tortricoid hosts (Lepidoptera, Tortricoidea). - Environ. Entomol. 21: 230-239.

Olesen, J. M. and Valido, A. 2003. Lizards as pollinators and seed dispersers: an island phenomenon. - Trends Ecol. Evol. 18: $177-181$

Olesen, J. M. and Valido, A. 2004. Lizards and birds as generalized pollinators and seed dispersers of island plants. - In: FernándezPalacios, J. M. and Morici, C. (eds), Ecología insular/Island ecology. Asociación Española de Ecología Terrestre (AEET) Cabildo Insular de La Palma, pp. 229-249.

Olesen, J. M. et al. 2002. Invasion of pollination networks on oceanic islands: importance of invader complexes and endemic super generalists. - Div. Distr. 8: 181-192.

Parent, C. E. and Crespi, B. J. 2009. Ecological opportunity in adaptive radiation of Galápagos endemic land snails. - Am. Nat. 174: 898-905.

Peck, S. B. et al. 1998. Introduced insect fauna of an oceanic archipelago: the Galápagos Islands, Ecuador. - Am. Entomol. 44: 218-237.

Supplementary material (available online as Appendix O19404 at <www.oikosoffice.lu.se/appendix >). Figure S1-S6.
Piechnik, D. A. et al. 2008. Food-web assembly during a classic biogeographic study: species' "trophic breadth" corresponds to colonization order. - Oikos 117: 665-674.

Quicke, D. L. J. 1997. Parasitic wasps. - Chapman and Hall.

Ribeiro, S. P. et al. 2005. Canopy insect herbivore diversity and distribution in the native forests of the Azores: key host plant species in a highly generalist insect community. - Ecography 28: 315-330.

Rodríguez, M. Á. and Hawkins, B. A. 2000. Diversity, function and stability in parasitoid communities. - Ecol. Lett. 3: 35-40.

Rosenzweig, M. L. 1995. Species diversity in space and time. Cambridge Univ. Press.

Santos, A. M. C. et al. 2005. Riqueza de espécies e diversidade ecológica de himenópteros parasitóides (Hymenoptera, Parasitica) em culturas frutícolas da ilha Terceira (Açores). - In: Lopes, D. et al. (eds.), A fruticultura na Macaronésia - o contributo do projecto INTERFRUTA para o seu desenvolvimento. Univ. dos Açores, pp. 137-151.

Santos, A. M. C. et al. 2011. Applying DNA barcoding for the study of geographical variation in host-parasitoid interactions. - Mol. Ecol. Resour. 11: 46-59.

Santos, A. M. C. et al. Species pool determines the level of generalism of island parasitoid faunas. - J. Biogeogr. in press.

Schlotfeldt, B. S. and Kleindorfer, S. 2006. Adaptive divergence in the superb fairy-wren (Malurus cyaneus): a mainland versus island comparison of morphology and foraging behaviour. Emu 106: 309-319.

Schoener, T. W. et al. 1995. Variation in the hymenopteran parasitoid fraction on Bahamian islands. - Acta Oecol. 16: 103-121.

Scott, S. N. et al. 2003. Morphological shifts in island-dwelling birds: the roles of generalist foraging and niche expansion. Evolution 57: 2147-2156.

Shaw, M. R. 1994. Parasitoid host range. - In: Hawkins, B. A. and Sheenan, W. (eds), Parasitoid community ecology. Oxford Univ. Press, pp. 111-144.

Sheehan, W. and Hawkins, B. A. 1991. Attack strategy as an indicator of host range in metopiine and plimpline Ichneumonidae (Hymenoptera). - Ecol. Entomol. 16: 129-131.

Stone, G. N. et al. 1995. Geographic and between-generation variation in the parasitoid communities associated with an invading gallwasp Andricus quercuscalicis. - Oecologia 104: 207-217.

Triantis, K. A. et al. 2010. The Macaronesian archipelago: patterns of species richness and endemism of arthropods. - In: Serrano, A. R. M. et al. (eds), Terrestrial arthropods of Macaronesia biodiversity ecology and evolution, pp. 45-64.

Tylianakis, J. M. et al. 2006. Diversity, ecosystem function and stability of parasitoid-host interactions across a tropical habitat gradient. - Ecology 87: 3047-3057.

van Nouhuys, S. and Hanski, I. 2002. Colonization rates and distances of a host butterfly and two specific parasitoids in a fragmented landscape. - J. Anim. Ecol. 71: 639-650.

Whittaker, R. J. and Fernández-Palacios, J. M. 2007. Island biogeography: ecology, evolution and conservation. - Oxford Univ. Press.

Williamson, M. 1981. Island populations. - Oxford Univ. Press. 